\title{
ORBITAL ULTRASOUND IN THE OCULAR ISCHAEMIC SYNDROME
}

\author{
PAUL RIORDAN-EVA ${ }^{1,3}$, MARIE RESTORI ${ }^{2}$, A. M. PETER HAMILTON ${ }^{1}$ and IVOR S. LEVY ${ }^{3}$ \\ London
}

\begin{abstract}
SUMMARY
The ocular ischaemic syndrome illustrates well the effects of hypoperfusion of the globe and is a useful model for studying disorders of the orbital circulation. Recent advances in orbital ultrasound techniques have provided a non-invasive method of examining flow velocities in the orbital vessels, particularly the ophthalmic artery. Orbital ultrasound studies were performed on 3 cases of the ocular ischaemic syndrome. Continuous or intermittent reversal of blood flow in the ophthalmic artery was seen in all cases. How this phenomenon relates to the symptoms and signs in affected patients, and the role of orbital ultrasound in the investigation of the ocular ischaemic syndrome, are discussed.
\end{abstract}

The ocular ischaemic syndrome is the clinical manifestation of chronic hypoperfusion of the globe. ${ }^{1-4}$ It is most commonly due to severe occlusive disease, either atherosclerosis or inflammatory arteritis, of the carotid or innominate arteries either at their origin from the arch of the aorta or in the neck. Affected patients are thus at risk of ischaemic cerebrovascular disease and frequently they have coronary artery or peripheral vascular disease. Thus the prognosis for life as well as for vision is threatened. Effective management requires early diagnosis and an easy, reliable method of monitoring disease progression.

This paper reports on the use of orbital ultrasound studies in the investigation of ophthalmic artery flow velocities in 3 dissimilar cases of the ocular ischaemic syndrome. It exemplifies the value of the technique in diagnosis, and the insights provided into changes in the circulation to the globe and brain that result from severe occlusive arterial disease in the extracranial vessels.

\section{PULSED ULTRASOUND STUDIES OF THE ORBIT}

The orbital blood vessels, except for the superior ophthal-

From: 'Retinal Diagnostic Department and ${ }^{2}$ Department of Ultrasound, Moorfields Eye Hospital, London; ${ }^{3}$ Department of Ophthalmology, The Royal London Hospital, London, UK.

Correspondence to: Mr P. Riordan-Eva, FRCS, FRCOphth, Department of Neuro-Ophthalmology, National Hospital for Neurology and Neurosurgery, Queen Square, London WC1N 3BG, UK. mic vein, are rarely identified with grey-scale B-mode ultrasound imaging. Application of newer techniques allows both the localisation of orbital blood vessels and the quantitative assessment of the velocity of blood flowing within them. Colour flow mapping uses pulsed ultrasound to detect the changing locations of red blood cells within a blood vessel during a short time period such that the velocity of blood flow can be determined electronically. ${ }^{5,6}$ This velocity information is colour coded and superimposed on to the B-mode image in real time to produce a colour flow image. ${ }^{7,8}$ The usual convention is to represent flow towards the transducer in red and flow away from the transducer in blue. Different velocities are represented as different hues of colour or as different colours. The normal ophthalmic artery is easily and rapidly identified on the colour image as a band of red shades flashing in time with the heart rate. The central retinal artery, central retinal vein, long and short posterior ciliary arteries, superior ophthalmic vein and vortex veins are also identifiable. ${ }^{7,8}$

Pulsed 'spectral Doppler' techniques are then applied to a selected region within the colour flow map to produce a graphical output of 'line of sight' velocity (dependent on the angle between the direction of interrogating pulses and the direction of blood flow) versus time. The brightness of the graphical display at any point is an indication of the number of blood cells exhibiting a particular velocity of flow. The usual convention is to show flow towards the transducer above the $x$-axis and flow away from the transducer below the $x$-axis. The normal ophthalmic arterial trace is characterised by a waveform above the $x$-axis with a maximum velocity which varies from the peak of systole through to the end of diastole. Maximum systolic blood flow velocity in the ophthalmic artery is normally $31.4 \pm 4.2$ (mean $\pm \mathrm{SD}) \mathrm{cm} / \mathrm{s}^{8}$

In this study orbital ultrasound was used to investigate direction and velocity of flow in the ophthalmic artery. Patients were examined in the sitting position with the Acuson 128 ultrasound machine using a $7 \mathrm{MHz}$ sector probe and a sample gate of $2 \mu \mathrm{s}$. Scanning was performed 
in the transverse plane with the probe directed at the superonasal quadrant of the orbit. When necessary the patient was asked to look downwards and laterally to facilitate identification of the ophthalmic artery. The central retinal artery and vein and the posterior ciliary vessels were not examined in these studies.

\section{CASE REPORTS}

\section{Case 1}

A 65-year-old man with a history of coronary artery disease presented with poor central vision in his left eye. Visual acuity was reduced to $6 / 18$ compared with $6 / 6$ in the right eye. Anterior segment examination was normal with equal intraocular pressures. The right fundus was normal. In the left fundus there was a cotton wool spot superonasal to the fovea and the retinal veins were dilated and irregular. Fluorescein angiography showed areas of retinal ischaemia at the macula with fluorescein leakage from their edges (Fig. 1). In the peripheral retina there were many microaneurysms and fluorescein leakage from the retinal vessels. The patient was treated with systemic steroids for a presumed retinal vasculitis without any benefit. The patient subsequently suffered a left hemisphere cerebrovascular accident. Soon afterwards iris rubeosis and a low central retinal artery perfusion pressure were noted in the left eye. The carotids were normal on examination but an intravenous digital subtraction angiogram (DSA) confirmed the presence of an occluded left internal carotid artery. Orbital ultrasound studies showed normalvelocity anterograde flow in the right ophthalmic artery and normal-velocity (peak systolic flow velocity $39 \mathrm{~cm} / \mathrm{s}$ ) retrograde flow in the left ophthalmic artery (Fig. 2).

The left eye subsequently developed more extensive retinal ischaemia followed by both optic disc and iris neo-

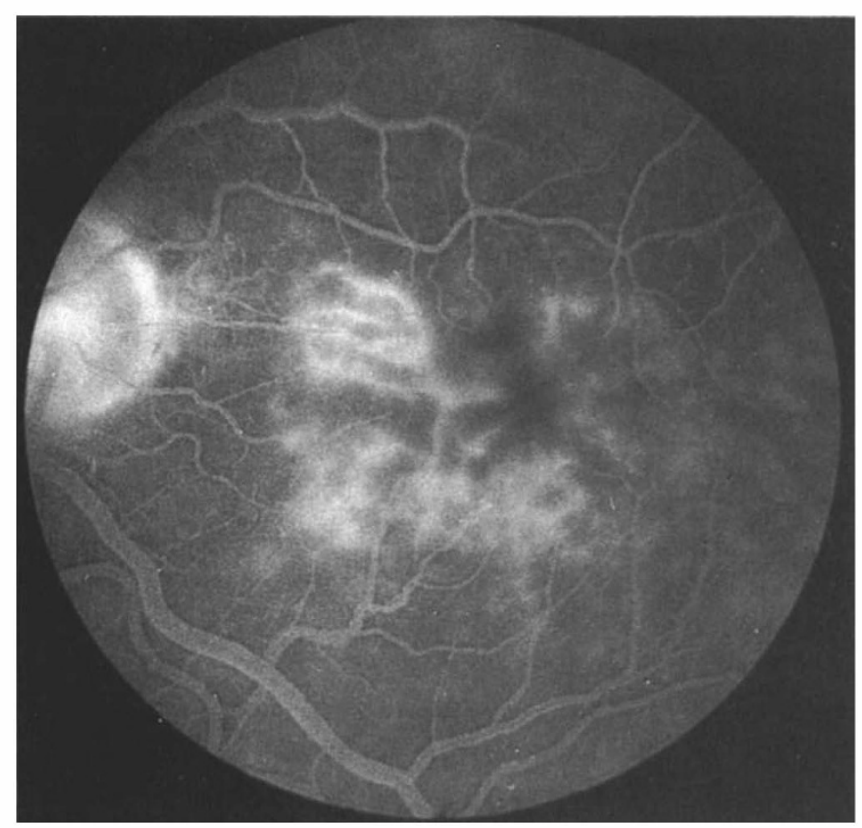

Fig. 1. Case 1. Late phase of fluorescein angiogram of left eye showing leakage from the edges of the ischaemic areas at the macula. vascularisation. Despite panretinal photocoagulation there was repeated vitreous haemorrhage and vision was reduced to perception of light only.

\section{Case 2}

A 45-year-old diabetic man with no visual complaints was referred for assessment of a presumed background diabetic retinopathy in his left eye. The right eye was amblyopic from a childhood esotropia. Three years prior to examination he had suffered episodes of visual loss precipitated by sudden standing, but these had resolved spontaneously. Visual acuities were $6 / 18$ in the right eye and $6 / 6$ in the left. There was a moderate right concomitant esotropia. Anterior segment examination was normal in each eye with equal intraocular pressures. There was a slow-flow retinopathy in the left eye manifesting as multiple mid-peripheral blot haemorrhages (Fig. 3) associated with irregular dilatation of retinal veins, and a central retinal artery that was easily occluded by pressure on the globe. The right fundus was normal without any diabetic retinopathy. Both carotid pulses were palpable without any bruits. Neurological examination and a CT brain scan were normal. Fluorescein angiography showed delayed choroidal filling and slow retinal arteriovenous transit time in the early phase, and mild macular oedema in the late phase.

Orbital ultrasound studies showed markedly reducedvelocity (peak systolic flow velocity $4 \mathrm{~cm} / \mathrm{s}$ ) anterograde flow in the right ophthalmic artery and high-velocity (peak systolic flow velocity $45 \mathrm{~cm} / \mathrm{s}$ ) retrograde flow in the left ophthalmic artery. Carotid angiography demonstrated complete occlusion of the origin of the left internal carotid artery (Fig. 4) and mild stenosis at the origin of the right internal carotid artery. On left common carotid injection the circle of Willis filled from the external carotid circulation by retrograde flow along the ophthalmic artery.

\section{Case 3}

A 53-year-old woman with familial hypercholesterol-

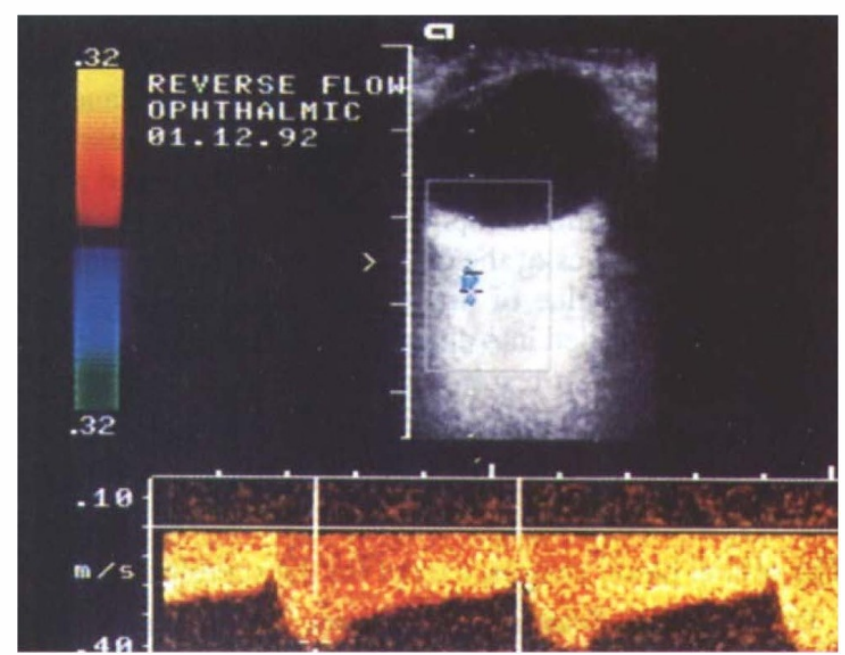

Fig. 2. Case 1. Left orbital ultrasound study showing reversal of flow in the ophthalmic artery. The blue colour on the colour flow image and the waveform below the $x$-axis on the spectral waveform trace indicate that blood is flowing away from the globe. 


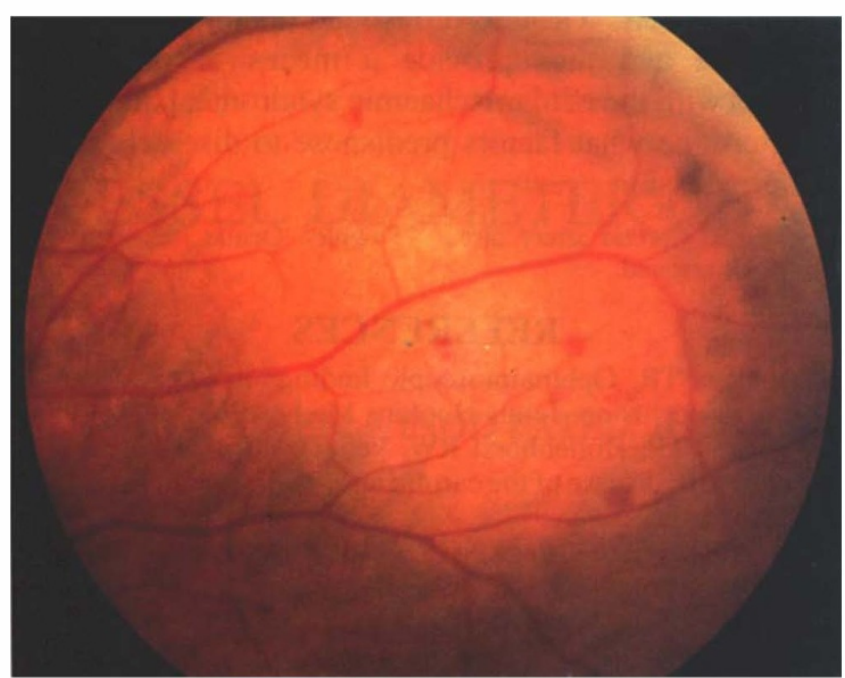

Fig. 3. Case 2. Left fundus photograph showing multiple midperipheral blot haemorrhages.

aemia was noted to have an absent right carotid pulse and a left carotid bruit during investigation for severe coronary artery disease. She admitted to episodes of visual loss, sometimes bilateral and sometimes unilateral, particularly precipitated by raising her arms or by standing up suddenly. These episodes were associated with a feeling of dizziness which sometimes progressed to a complete blackout with unconsciousness. Visual acuities were 6/9 in each eye. Ophthalmic examination was otherwise unremarkable apart from precipitation of visual loss by pressure on the ipsilateral globe. There were no abnormal neurological signs. The right radial pulse and right brachial blood pressure were reduced. Orbital ultrasound studies showed normal ophthalmic artery flow direction in each eye with the arms lowered. Raising the arms above the horizontal produced reversal of flow in both ophthalmic arteries.

Angiography demonstrated occlusion of the innominate artery at its origin, a tight stenosis at the right carotid bifurcation, $80 \%$ stenosis of the left internal carotid and $75 \%$ stenosis at the origin of the left subclavian.

\section{DISCUSSION}

The ocular ischaemic syndrome is frequently the only clinical manifestation of severe occlusive disease of the carotid or innominate arteries. ${ }^{3}$ Its recognition is hampered by the similarity of the resultant visual obscurations to amaurosis fugax due to retinal emboli, the similarity of slow-flow retinopathy to diabetic retinopathy or retinal vein occlusion, and the frequent absence of neurological symptoms or signs. ${ }^{2,3,9}$ But establishing the diagnosis is essential to the correct management of the patient and this needs to be done with minimum risk to the patient.

Ophthalmodynanometry and fluorescein angiography demonstrate the ocular hypoperfusion but the former is relatively insensitive and inaccurate and the latter is difficult to quantify. Identification of the underlying arterial disease can be undertaken by angiography, but this is expensive, time-consuming, and may harm the patient either through adverse reactions to contrast agents, vascular spasm, or high levels of irradiation if repeated angiograms are used to monitor disease progression.

Ultrasound is acquiring an increasingly important role in the assessment of both extracranial and intracranial vascular disease. Carotid Doppler studies are an accepted method of investigating occlusive carotid disease and their reliability and accuracy are continually being improved. ${ }^{10}$ Transcranial Doppler allows for assessment of intracranial arterial flow velocities, particularly in the middle cerebral arteries. ${ }^{11}$ The adaptation of ultrasound colour flow imaging to the orbit offers a quick, non-invasive and easily repeatable measure of the dynamic state of the orbital circulation. ${ }^{7,8}$ Vascular disorders to which orbital colour flow mapping has already been applied include acute and chronic occlusive carotid artery disease, ${ }^{12,13}$ central retinal artery occlusions and anterior ischaemic optic neuropathy, ${ }^{14}$ and carotid-cavernous fistulae. ${ }^{7,15}$

Reversal of flow in the ophthalmic artery is well recognised, particularly by radiologists, as a feature of internal carotid artery occlusion, ${ }^{16}$ being the consequence of the development of a collateral circulation from branches of the external carotid artery to the circle of Willis. The haemodynamic role of this collateral circulation with respect to the intracranial circulation is much debated. Both angiographic and ultrasound studies have suggested that

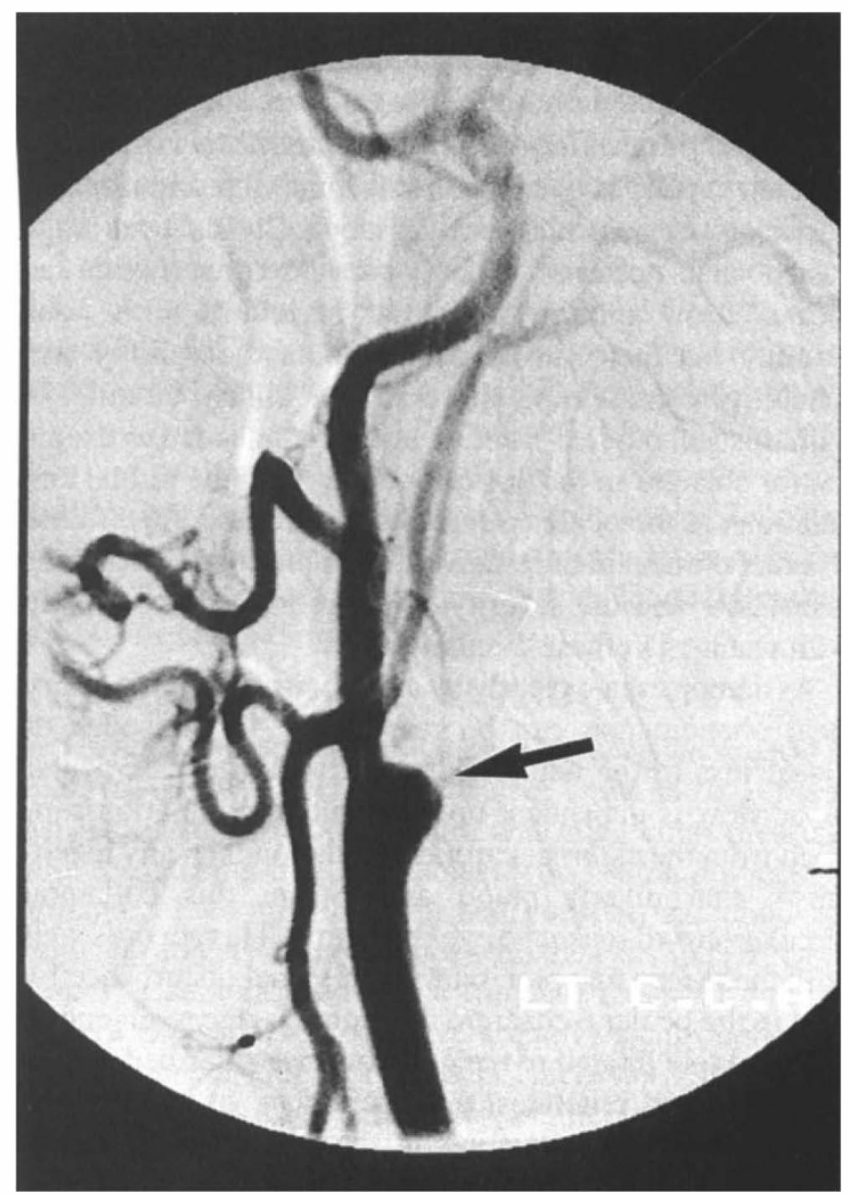

Fig. 4. Case 2. Left carotid angiogram showing occluded internal carotid artery (arrow). 
ophthalmic artery flow reversal occurs when there is inadequate crossflow in the circle of Willis from the contralateral internal carotid. ${ }^{16-18}$ In this respect it is interesting that in case 3, in which there was the most severe arterial disease, ophthalmic artery flow reversal was not a constant feature but was precipitated by raising the arms, whereas in case 1 , in which the arterial disease was limited to one internal carotid, the flow reversal was constant. This underlines the dynamic nature of the circulation and the advantage of ultrasound over angiography in understanding how the circulation adapts to different circumstances.

The role of ophthalmic artery flow reversal in the pathogenesis of the ocular ischaemic syndrome was first discussed in 1972 when it was suggested that an ophthalmic artery 'steal' phenomenon was the cause of the rubeosis iridis in 2 patients with internal carotid artery occlusion. ${ }^{19}$ But case 2 of this report demonstrates that ophthalmic artery flow reversal may be found in mild cases of the ocular ischaemic syndrome without rubeosis iridis. A previous study of orbital colour flow mapping in which ophthalmic artery flow reversal was demonstrated in 12 of 16 eyes with the ocular ischaemic syndrome secondary to occlusive carotid artery disease, found no correlation between the ophthalmic artery flow direction and the severity of the clinical signs. ${ }^{13}$ It is unclear what factors determine the severity and rate of progression of ocular ischaemia in patients with severe occlusive carotid artery disease. Only $4-18 \%$ of patients with internal carotid artery occlusion develop ocular ischaemia ${ }^{4}$ and the natural history in affected eyes is highly variable. Some eyes (case 2) remain only mildly affected whereas others (case 1) develop rapidly progressive ischaemia with anterior and posterior segment neovascularisation. It has been suggested that posterior ciliary artery hypoperfusion as measured by orbital ultrasound correlates with poor vision, ${ }^{13}$ but there is still debate about the validity and reliability of assessing the posterior ciliary circulation with this technique..$^{20}$ Further studies are needed to examine for changes in the posterior ciliary and the retinal circulations in the ocular ischaemic syndrome to determine whether clinical manifestations, particularly the degree of visual loss and the severity of the retinopathy, correlate with changes in these circulations.

As demonstrated clearly by case 3 , an ophthalmic artery steal phenomenon can be responsible for episodes of visual loss in the ocular ischaemic syndrome. If ocular blood flow is dependent upon collateral circulation supplied from the external carotid circulation then any mechanism that diverts blood away from this collateral circulation will make the eye ischaemic. This accords well with the theory that precipitation of visual disturbance by heat in the ocular ischaemic syndrome is due to diversion of blood to a dilated external carotid vascular bed. ${ }^{3}$

This paper reiterates the usefulness of ophthalmic artery colour flow imaging in the investigation of the ocular ischaemic syndrome, both for diagnosis and for furthering our understanding of the changes in the orbital circulation with severe occlusive disease of the extra- cranial vessels. Orbital ultrasound studies are easily repeatable and thus provide a means of monitoring patients with the ocular ischaemic syndrome, particularly to determine what factors predispose to disease severity and progression.

Key words: Carotid artery disease, Doppler, Ocular ischaemic syndrome, Ultrasound

\section{REFERENCES}

1. Hedges TR. Ophthalmoscopic findings in internal carotid artery occlusion. Johns Hopkins Med J 1962;111:89-97.

2. Kearns TP, Hollenhorst RW. Venous-stasis retinopathy of occlusive disease of the carotid artery. Mayo Clin Proc 1963; 38:304-12

3. Ross Russell RW, Page NGR. Critical perfusion of brain and retina. Brain 1983;106:419-34.

4. Sturrock GD, Mueller HR. Chronic ocular ischaemia. Br J Ophthalmol 1984;68:716-23.

5. Canning CR, Restori M. Doppler ultrasound studies of the ophthalmic artery. Eye 1988;2:92-5.

6. Taylor KJW, Holland S. Doppler US. I. Basic principles, instrumentation, and pitfalls. Radiology 1990;174:297-307.

7. Erickson SJ, Hendrix LE, Massaro BM, Harris GJ, Lewandowski MF, Foley WD, Lawson TL. Color Doppler flow imaging of the normal and abnormal orbit. Radiology 1989; 173:511-6.

8. Lieb WE, Cohen SM, Merton DA, Shields JA, Mitchell DG, Goldberg BB. Color Doppler imaging of the eye and orbit: technique and normal vascular anatomy. Arch Ophthalmol 1991;109:527-31.

9. Kearns TP. Differential diagnosis of central retinal vein occlusion. Ophthalmology 1983;90:475-80.

10. Scoutt LM, Zawin ML, Taylor KJW. Doppler US. II. Clinical applications. Radiology 1990;174:309-19.

11. Schneider PA, Rossman ME, Bernstein EF, Torem S, Ringelstein EB, Otis SM. Effect of internal carotid artery occlusion on intracranial hemodynamics: transcranial Doppler evaluation and clinical correlation. Stroke 1988;19:589-93.

12. Lieb WE, Flaharty PM, Sergott RC, Medlock RD, Brown GC, Bosley T, Savino PJ. Color Doppler imaging provides accurate assessment of orbital blood flow in occlusive carotid artery disease. Ophthalmology 1991;98:548-52.

13. Ho AC, Lieb WE, Flaharty PM, Sergott RC, Brown GC, Bosley TM, Savino PJ. Color Doppler imaging of the ocular ischaemic syndrome. Ophthalmology 1992;99:1453-62.

14. Williamson TH, Baxter GM, Dutton GN. Color Doppler velocimetry of the optic nerve head in arterial occlusion. Ophthalmology 1993;100:312-7.

15. Flaharty PM, Lieb WE, Sergott RC, Bosley TM, Savino PJ. Color Doppler imaging: a new noninvasive technique to diagnose and monitor carotid cavernous sinus fistulas. Arch Ophthalmol 1991;109:522-6.

16. Pitts FW. Variations of collateral circulation in internal carotid occlusion. Neurology 1962;12:469-71.

17. Hodek-Demarin V, Muller HR. Reversed ophthalmic artery flow in internal carotid artery occlusion: a re-appraisal based on ultrasonic Doppler investigations. Stroke 1979;10: 461-3.

18. Tatemichi TK, Chamorro A, Petty GW, Khandji A, Oropeza LA, Duterte DI, Mohr JP. Hemodynamic role of ophthalmic artery collateral in internal carotid artery occlusion. Neurology 1990;40:461-4.

19. Huckman MS, Haas J. Reversed flow through the ophthalmic artery as a cause of rubeosis iridis. Am J Ophthalmol 1972;74:1094-9.

20. Williamson TH, Baxter GM, Dutton GN. Colour Doppler velocimetry of the arterial vasculature of the optic nerve head and orbit. Eye 1993;7:74-9. 